\title{
Probing lepton universality with (semi)-leptonic $B$ decays
}

\author{
Giovanni Banelli ${ }^{1}$, Robert Fleischer ${ }^{1,2}$, \\ Ruben Jaarsma ${ }^{1}$ and Gilberto Tetlalmatzi-Xolocotzi ${ }^{1 \text { * }}$
}

1 Nikhef, Science Park 105, NL-1098 XG Amsterdam, Netherlands

2 Faculty of Science, Vrije Universiteit Amsterdam, NL-1081 HV Amsterdam, Netherlands

$\star$ gtx@nikhef.nl

Proceedings for the 15th International Workshop on Tau Lepton Physics, Amsterdam, The Netherlands, 24-28 September 2018

doi:10.21468/SciPostPhysProc. 1

\begin{abstract}
The most recent measurements of the observables $R_{D^{(*)}}$ are in tension with the Standard Model offering hints of New Physics in $b \rightarrow c \ell \bar{v}_{\ell}$ transitions. Motivated by these results, in this work we present an analysis on their $b \rightarrow u \ell \bar{v}_{\ell}$ counterparts (for $\ell=e, \mu, \tau$ ). Our study has three main objectives. Firstly, using ratios of branching fractions, we assess the effects of beyond the Standard Model scalar and pseudoscalar particles in leptonic and semileptonic $B$ decays $\left(B^{-} \rightarrow \ell^{-} \bar{v}_{\ell}, \bar{B} \rightarrow \pi \ell \bar{v}_{\ell}\right.$ and $\left.\bar{B} \rightarrow \rho \ell \bar{v}_{\ell}\right)$. Here a key role is played by the leptonic $B$ processes, which are highly sensitive to new pseudoscalar interactions. In particular, we take advantage of the most recent measurement of the branching fraction of the channel $B^{-} \rightarrow \mu^{-} \bar{v}_{\mu}$ by the Belle collaboration. Secondly, we extract the CKM matrix element $\left|V_{u b}\right|$ while accounting simultaneously for New Physics contributions. Finally, we provide predictions for the branching fractions of yet unmeasured leptonic and semileptonic $B$ decays.
\end{abstract}

(c) (1) Copyright G. Banelli et al.

This work is licensed under the Creative Commons

Attribution 4.0 International License.

Published by the SciPost Foundation.
Received 13-12-2018

Accepted 17-01-2019

Published 19-02-2019

doi:10.21468/SciPostPhysProc.1.013

\section{Contents}

1 Introduction $\quad 1$

2 Constraints from leptonic $B$ decays $\quad 3$

3 Semileptonic $B$ decays $\quad 4$

4 Determination of $\left|V_{u b}\right|$ and predictions of the branching fractions for $B^{-} \rightarrow e^{-} \bar{v}_{e}$ and $\bar{B} \rightarrow \rho \tau^{-} \bar{v}_{\tau}$

5 Outlook 


\section{Introduction}

Recent measurements of the observables $R_{D^{(*)}} \equiv \mathcal{B}\left(B \rightarrow D^{(*)} \tau \bar{v}_{\tau}\right) / \mathcal{B}\left(B \rightarrow D^{(*)} \ell^{\prime} \bar{v}_{\tau}\right)$, with $\ell^{\prime}=e, \mu$, have caused a lot of excitement in the high-energy physics community. As a matter of fact, the combined measurements of the BaBar, LHCb and Belle collaborations show a $3.9 \sigma$ deviation with respect to the expected value from the Standard Model (SM) [1]. If this effect is confirmed by forthcoming experimental determinations, it will indicate the presence of New Physics (NP) in the exclusive decays $\bar{B} \rightarrow D^{(*)} \tau \bar{v}_{\tau}$, which are caused by the quarklevel transition $b \rightarrow c \tau \bar{v}_{\tau}$ [2]. Motivated by these results, we investigate the presence of NP effects in $b \rightarrow u \ell \bar{v}_{\ell}$ processes, where $\ell=e, \mu, \tau$. To derive the relevant constraints, our study involves the interplay of the leptonic decay channels $B^{-} \rightarrow \ell^{-} \bar{v}_{\ell}$ with the semileptonic transitions $\bar{B} \rightarrow \pi \ell \bar{v}_{\ell}$ (analogous to $\bar{B} \rightarrow D \ell \bar{v}_{\ell}$ ) and $\bar{B} \rightarrow \rho \ell \bar{v}_{\ell}$ (analogous to $\bar{B} \rightarrow D^{*} \ell \bar{v}_{\ell}$ ).

The different decays to be included in this study are sensitive to NP scalar, pseudoscalar, vector and tensor interactions $[3,4]$. The pseudoscalar components are special because, due to the structure of the equations for the branching fractions $\mathcal{B}\left(B^{-} \rightarrow \ell^{-} \bar{v}_{\ell}\right)$, they lift the helicity suppression appearing in the corresponding SM expressions. This effect leads to interesting phenomenological predictions. Therefore, we focus on the pseudoscalar contributions and complement our NP analysis by including also scalar operators, which are their natural partners in terms of the Lorenz structure.

We follow an effective theory approach, with the low-energy Hamiltonian

$$
\mathcal{H}_{\mathrm{eff}}=\frac{4 G_{\mathrm{F}}}{\sqrt{2}} V_{u b}\left[C_{V_{L}} \mathcal{O}_{V_{L}}^{\ell}+C_{S}^{\ell} \mathcal{O}_{S}^{\ell}+C_{P}^{\ell} \mathcal{O}_{P}^{\ell}\right]+\text { h.c., }
$$

where

$$
\mathcal{O}_{V_{L}}^{\ell}=\left(\bar{q} \gamma^{\mu} P_{L} b\right)\left(\bar{\ell} \gamma_{\mu} P_{L} v_{\ell}\right), \quad \mathcal{O}_{S}^{\ell}=(\bar{q} b)\left(\bar{\ell} P_{L} v_{\ell}\right), \quad \mathcal{O}_{P}^{\ell}=\left(\bar{q} \gamma_{5} b\right)\left(\bar{\ell} P_{L} v_{\ell}\right)
$$

are the corresponding vector, scalar and pseudoscalar operators, respectively. The shortdistance contributions are encoded in the Wilson coefficients $C_{V_{L}}, C_{S}^{\ell}$ and $C_{P}^{\ell}$. In the SM, only $C_{V_{L}}$ is non-vanishing and takes the value $C_{V_{L}}=1$. However, $C_{S}^{\ell}$ and $C_{P}^{\ell}$ may be non-zero in NP scenarios. An important example is the Two Higgs Doublet Model (2HDM) [3], where the scalar and pseudoscalar coefficients are related to each other as follows:

$$
C_{S}^{\ell}=C_{P}^{\ell}=-\tan ^{2} \beta\left(\frac{m_{b} m_{l}}{M_{H^{ \pm}}^{2}}\right)
$$

In general, the Wilson coefficients in Eq. (1) can be complex, with CP-violating NP phases. Unfortunately, direct CP asymmetries associated with leptonic and semileptonic processes cannot be used in their determination, because they vanish due to the absence of sizeable CP conserving phase differences. Hence, as discussed in Ref. [5], in order to search for new sources of CP violation, a different strategy based on correlations between magnitudes and phases of Wilson coefficients should be followed. For simplicity, our discussion in this report will be limited to real $C_{S}^{\ell}$ and $C_{P}^{\ell}$.

It is important to bear in mind that the exclusive determination of the CKM matrix element $\left|V_{u b}\right|$ is done using semileptonic $B$ decays originating from the quark level transition $b \rightarrow u \ell \bar{v}_{\ell}$ assuming the SM. However, this value may be affected by NP effects. Therefore, in order to take into account these contributions we propose the following strategy: 
1. Using combinations of ratios of branching fractions for leptonic and semileptonic $B$ decays, where $\left|V_{u b}\right|$ cancels, we determine the allowed regions for $C_{S}^{\ell}$ and $C_{P}^{\ell}$.

2. We then use these numerical ranges for $C_{S}^{\ell}$ and $C_{P}^{\ell}$ and evaluate the branching fraction for given leptonic or semileptonic $B$ processes, allowing us to finally extract $\left|V_{u b}\right|$ from data.

In the following sections, we will elaborate on this procedure and we will present our predictions for the branching fractions of leptonic and semileptonic $B$ decays which have not yet been measured. The full study can be found in Ref. [5].

\section{Constraints from leptonic $B$ decays}

In the SM, the branching fraction of the process $B^{-} \rightarrow \ell^{-} \bar{v}_{\ell}$ is given by

$$
\left.\mathcal{B}\left(B^{-} \rightarrow \ell^{-} \bar{v}_{\ell}\right)\right|_{\mathrm{SM}}=\frac{G_{\mathrm{F}}^{2}}{8 \pi}\left|V_{u b}\right|^{2} M_{B^{-}} m_{\ell}^{2}\left(1-\frac{m_{\ell}^{2}}{M_{B^{-}}^{2}}\right)^{2} f_{B^{-}}^{2} \tau_{B^{-}} .
$$

We observe that the branching ratio is proportional to the square of the mass of the lepton in the final state $m_{\ell}^{2}$. For electrons and muons, this factor very strongly suppresses the decay probabilities, which is referred as helicity suppression. Interestingly, it is also present in the rare decays $B_{d, s} \rightarrow \ell^{+} \ell^{-}$[6]. Since $m_{e}, m_{\mu} \ll m_{\tau}$, the helicity suppression is less effective for tau leptons.

Leptonic $B$ decays are very clean channels, where all the non-perturbative hadronic information is encoded in the decay constant given by $[7,8]$

$$
f_{B^{-}}=0.186 \pm 0.004 \text {. }
$$

Assuming the SM, we use the CKMFitter value [9]

$$
\left|V_{u b}\right|=(3.601 \pm 0.098) \times 10^{-3},
$$

together with Eq. (6) to obtain

$$
\begin{aligned}
\mathcal{B}\left(B^{-} \rightarrow \tau^{-} \bar{v}_{\tau}\right) & =(7.92 \pm 0.55) \times 10^{-5}, \\
\mathcal{B}\left(B^{-} \rightarrow \mu^{-} \bar{v}_{\mu}\right) & =(3.56 \pm 0.25) \times 10^{-7}, \\
\mathcal{B}\left(B^{-} \rightarrow e^{-} \bar{v}_{e}\right) & =(8.33 \pm 0.58) \times 10^{-12},
\end{aligned}
$$

where due the tiny value of the mass of the electron, the helicity suppression leads to a extremely small branching fraction for the channel $B^{-} \rightarrow e^{-} \bar{v}_{e}$.

The experimental results reported by BaBar, Belle and LHCb lead to

$$
\begin{aligned}
\mathcal{B}\left(B^{-} \rightarrow \tau^{-} \bar{v}_{\tau}\right) & =(1.09 \pm 0.24) \times 10^{-4},[10] \\
\mathcal{B}\left(B^{-} \rightarrow \mu^{-} \bar{v}_{\mu}\right) & =(6.46 \pm 2.74) \times 10^{-7},[11] \\
\mathcal{B}\left(B^{-} \rightarrow e^{-} \bar{v}_{e}\right) & <9.8 \times 10^{-7}(90 \% \text { C.L. })[12] .
\end{aligned}
$$

Here the measurement corresponding to the channel $B^{-} \rightarrow \mu^{-} \bar{v}_{\mu}$ was reported recently by the Belle collaboration with a $2.4 \sigma$ excess over background and will be key during our phenomenological study. 


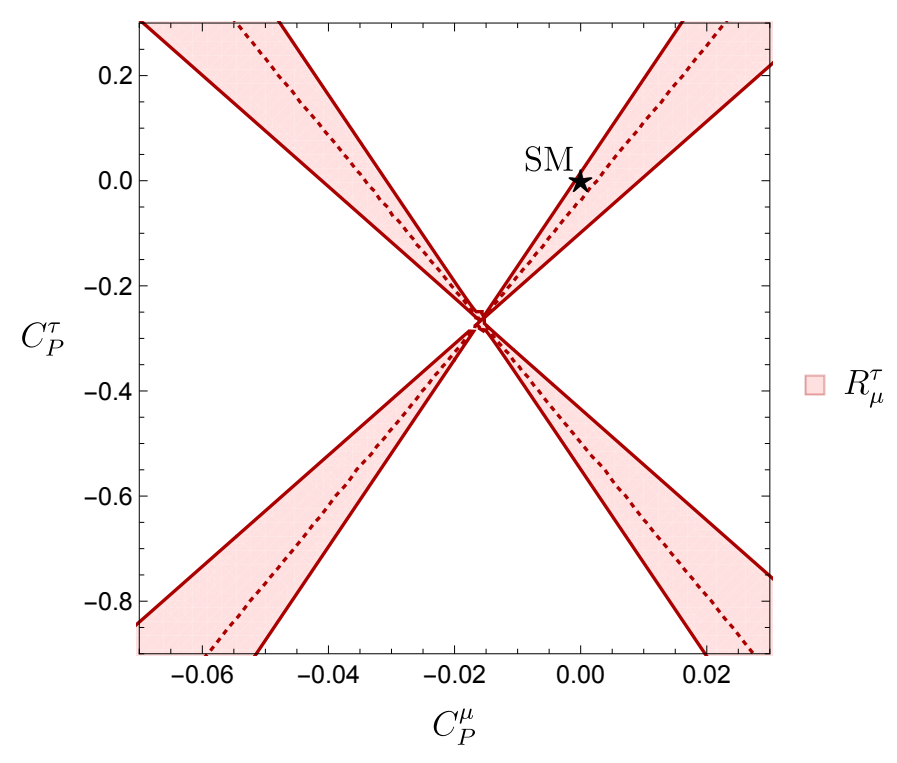

Figure 1: Allowed regions in the $C_{P}^{\mu}-C_{P}^{\tau}$ plane following from the leptonic ratio $R_{\mu}^{\tau}$.

For leptonic $B$ decays, the hadronic matrix element of the $O_{S}$ operator in Eq. (1) vanishes. Consequently, is does not receive scalar NP contributions. Once pseudoscalar NP effects are taken into account, Eq. (4) gets modified as

$$
\mathcal{B}\left(B^{-} \rightarrow \ell^{-} \bar{v}_{\ell}\right)=\left.\mathcal{B}\left(B^{-} \rightarrow \ell^{-} \bar{v}_{\ell}\right)\right|_{\mathrm{SM}}\left|1+\frac{M_{B^{-}}^{2}}{m_{\ell}\left(m_{b}+m_{u}\right)} C_{P}^{\ell}\right|^{2} .
$$

In the case of electrons and muons, the helicity suppression is lifted by the mass ratio

$$
M_{B^{-}}^{2} /\left[m_{\ell}\left(m_{b}+m_{u}\right)\right] \sim M_{B^{-}} / m_{\ell},
$$

thereby amplifying the effects of $C_{P}^{\ell}$.

To constrain the pseudoscalar Wilson coefficients $C_{P}^{\mu}$ and $C_{P}^{\tau}$, we consider the observable

$$
R_{\mu}^{\tau} \propto \frac{\mathcal{B}\left(B^{-} \rightarrow \tau^{-} \bar{v}_{\tau}\right)}{\mathcal{B}\left(B^{-} \rightarrow \mu^{-} \bar{v}_{\mu}\right)}
$$

where the normalization factor is chosen in such a way that we get $R_{\mu}^{\tau}=1$ in the SM. The main features of $R_{\mu}^{\tau}$ are the cancellation of the hadronic decay constant $f_{B^{-}}$and of the CKM matrix element $\left|V_{u b}\right|$. By comparing the corresponding theoretical determination for this ratio with the experimental result, we obtain the regions shown in Fig. 1. Here we can see that, even though $R_{\mu}^{\tau}$ is already imposing strong constraints on the values that $C_{P}^{\mu}$ and $C_{P}^{\tau}$ can take, the arms of the resulting cross-shaped area extend to infinity. To improve our bounds on these pseudoscalar Wilson coefficients, we have to include more observables sensitive to $C_{P}^{\mu}$ and $C_{P}^{\tau}$. This topic will be discussed in the next section. 


\section{Semileptonic $B$ decays}

To derive stronger constraints on $C_{P}^{\mu}$ and $C_{P}^{\tau}$, we consider the branching ratio $\mathcal{B}\left(\bar{B} \rightarrow \rho \ell \bar{v}_{\ell}\right)$, which in analogy with the leptonic decays, does not depend on scalar NP contributions [13]. Due to the presence of the $\rho$ meson in the final state, the hadronic contributions are more complicated than those encountered in the leptonic $B$ decays. In the helicity basis, these effects are encoded in the form factors denoted as $H_{V,+}^{\rho}, H_{V,-}^{\rho}, H_{V, 0}^{\rho}, H_{V, t}^{\rho}$ and $H_{S}^{\rho}$. The nonperturbative technique employed for their calculation depends on the value of the square of the four-momentum transferred $q^{2}$ to $\ell$ and $\bar{v}_{\ell}$. In the literature, two main approaches are usually considered for their determination:

- QCD sum rules for the low energy regime $0 \leq q^{2} \leq q_{\max }^{2}$, where typically $q_{\max }^{2} \in[12,16] \mathrm{GeV}^{2}$.

- Lattice QCD calculations $[14,15]$ are applied when $q^{2}$ is close to the maximal leptonic momentum transfer: $q_{\max }^{2} \leq q^{2} \leq\left(M_{B}-M_{\rho}\right)^{2}$.

As experimental input we consider the measurements of $\mathcal{B}\left(\bar{B}^{0} \rightarrow \rho^{+} \ell^{-} \bar{v}_{\ell}\right)$ and $\mathcal{B}\left(B^{-} \rightarrow \rho^{0} \ell^{-} \bar{v}_{\ell}\right)$ reported by Belle in 2013, which include an admixture of electrons and muons in the final state [13]. Using the isospin symmetry, we combine these two measurements to obtain $\left\langle\mathcal{B}\left(\bar{B} \rightarrow \rho \ell^{-} \bar{v}_{\ell}\right)\right\rangle_{[\ell=e, \mu], q^{2} \leq 12 \mathrm{GeV}^{2}}=(1.98 \pm 0.12) \times 10^{-4}$. We introduce the ratio

$$
\mathcal{R}_{\langle e, \mu\rangle ; \rho\left[q^{2} \leq 12\right] \mathrm{GeV}^{2}}^{\mu} \equiv \mathcal{B}\left(B^{-} \rightarrow \mu^{-} \bar{v}\right) /\left\langle\mathcal{B}\left(\bar{B} \rightarrow \rho \ell^{-} \bar{v}_{\ell}\right)\right\rangle_{[\ell=e, \mu], q^{2} \leq 12 \mathrm{GeV}^{2}},
$$

where the CKM element $\left|V_{u b}\right|$ cancels.

Since the experimental determinations do not yet provide independent information for electrons and muons, we can only obtain the allowed values for $C_{P}^{e}$ and $C_{P}^{\mu}$ if we correlate these Wilson coefficients through different assumptions. We start by testing the hypothesis of having universal NP interactions in electrons and muons, i.e. $C_{P}^{e}=C_{P}^{\mu}$, and explore the behaviour of the semileptonic decay in the range $q^{2} \leq 12 \mathrm{GeV}^{2}$, where an analytical parameterization from QCD sum rules is available [16]. Then, we proceed to determine the allowed regions in the $C_{P}^{\mu}-C_{P}^{\tau}$ plane by using the observables $R_{\mu}^{\tau}$ and $\mathcal{R}_{\langle e, \mu\rangle ; \rho\left[q^{2} \leq 12\right] \mathrm{GeV}^{2}}^{\mu}$ as constraints. Moreover, we include the leptonic ratio $R_{\mu}^{e}$, which is analogous to $R_{\mu}^{\tau}$ in Eq. (11), calculated from the experimental bound available for $\mathcal{B}\left(B^{-} \rightarrow e^{-} \bar{v}_{e}\right)$ presented in Eq. (8). The resulting plot is shown in Fig. 2, where only the elliptical areas labelled as "1" and "2" are allowed. Even though solution " 1 " is compatible with the SM, the solution inside region " 2 ", corresponding to NP, is not excluded.

So far, our treatment has been focused on constraining the pseudoscalar Wilson coefficient $C_{P}^{\ell}$. In order to have sensitivity on the scalar Wilson coefficient $C_{S}^{\ell}$, we include the branching ratios of the processes $\bar{B} \rightarrow \pi \ell \bar{v}_{\ell}$. Unfortunately, the current experimental information does not provide independent measurements for electrons and muons. To incorporate these processes in our analysis, we make an average using the isospin symmetry to combine the independent contributions of $B^{0}$ and $B^{-}$provided in Ref. [10], yielding $\left\langle\mathcal{B}\left(\bar{B} \rightarrow \pi \ell \bar{v}_{\ell}\right)\right\rangle_{[\ell=e, \mu]}=(1.53 \pm 0.04) \times 10^{-4}$.

Two more observables, which are sensitive to both $C_{S}^{\ell}$ and $C_{P}^{\ell}$, are now at our disposal: 


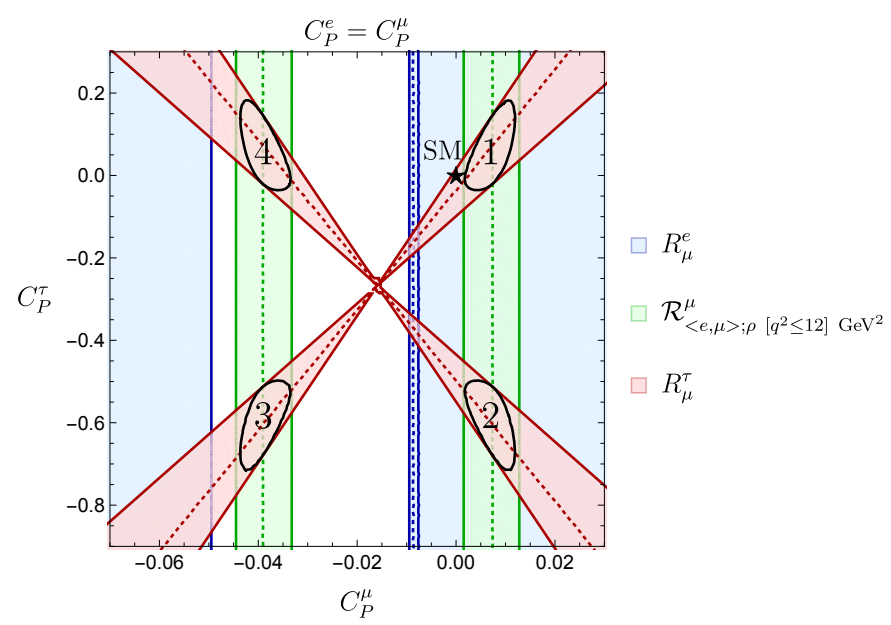

Figure 2: Allowed regions in the $C_{P}^{\mu}-C_{P}^{\tau}$ plane utilizing the ratios $R_{\mu}^{e}, R_{\mu}^{\tau}$ and $\mathcal{R}_{\langle e, \mu\rangle ; \rho\left[q^{2} \leq 12\right] \mathrm{GeV}^{2}}^{\mu}$ under the assumption $C_{P}^{e}=C_{P}^{\mu}$.

$$
\begin{gathered}
\mathcal{R}_{\langle e, \mu\rangle ; \pi}^{\mu} \equiv \mathcal{B}\left(B^{-} \rightarrow \mu^{-} \bar{v}\right) /\left\langle\mathcal{B}\left(\bar{B} \rightarrow \pi \ell^{-} \bar{v}_{\ell}\right)\right\rangle \\
\left.\mathcal{R}_{\langle e, \mu\rangle ; \pi}^{\left.\langle e, \mu\rangle ; q_{\min }^{2} \leq q^{2} \leq q_{\max }^{2}\right]} \equiv\left\langle\mathcal{B}\left(\bar{B} \rightarrow \rho \ell^{-} \bar{v}_{\ell}\right)\right\rangle_{[\ell=e, \mu]}\right|_{q_{\min }^{2}} ^{q_{\max }^{2}} /\left\langle\mathcal{B}\left(\bar{B} \rightarrow \pi \ell^{-} \bar{v}_{\ell}\right)\right\rangle_{[\ell=e, \mu]}
\end{gathered}
$$

Including furthermore $\mathcal{R}_{\langle e, \mu\rangle ; \rho\left[q^{2} \leq 12\right] \mathrm{GeV}^{2}}^{\mu}$, introduced in Eq. (12), and making the assumptions $C_{P}^{e}=C_{P}^{\mu}, C_{S}^{e}=C_{S}^{\mu}$, we obtain the regions in the $C_{S}^{\mu}-C_{P}^{\mu}$ plane shown in Fig. 3. We observe that $\mathcal{R}_{\langle e, \mu\rangle ; \pi}^{\langle e, \mu\rangle ; \rho\left[0 \leq q^{2} \leq 12\right] \mathrm{GeV}^{2}}$ results in two horizontal bands, which are in tension with the SM at $(1-2) \sigma$.

We have restricted our studies to the low $q^{2}$ regime. Let us now investigate whether the tension found with the SM persists for large $q^{2}$ values. To the best of our knowledge, the only available hadronic inputs for $B \rightarrow \rho$ transitions in the $12 \mathrm{GeV}^{2} \leq q^{2}$ range come from lattice determinations [15] and were obtained in 2004. Due to the unstable nature of the $\rho$ meson, these calculations are rather challenging. To keep the non-perturbative uncertainties under control when evaluating the branching ratios, we use differential distributions in $q^{2}$ rather than fully integrated expressions. Therefore, for $12 \mathrm{GeV}^{2}<q^{2}$ we introduce the following observable:

$$
d \mathcal{R}_{\langle e, \mu\rangle ; \pi}^{\langle e, \mu\rangle ; \rho}=\frac{2\left\langle d \mathcal{B}\left(B^{-} \rightarrow \rho^{0} \ell^{-} \bar{v}_{\ell}\right) / d q^{2}\right\rangle_{[\ell=e, \mu]}}{\left\langle\mathcal{B}\left(\bar{B} \rightarrow \pi \ell^{-} \bar{v}_{\ell}\right)\right\rangle_{[\ell=e, \mu]}}
$$

Interestingly, as can be seen in Fig. 4, we find a mild tension with the SM for $q^{2}=17 \mathrm{GeV}^{2}$. To shed light on the origin of this feature, an updated determination of the form factors for the transition $B \rightarrow \rho$ in the high $q^{2}$ regime is needed. 


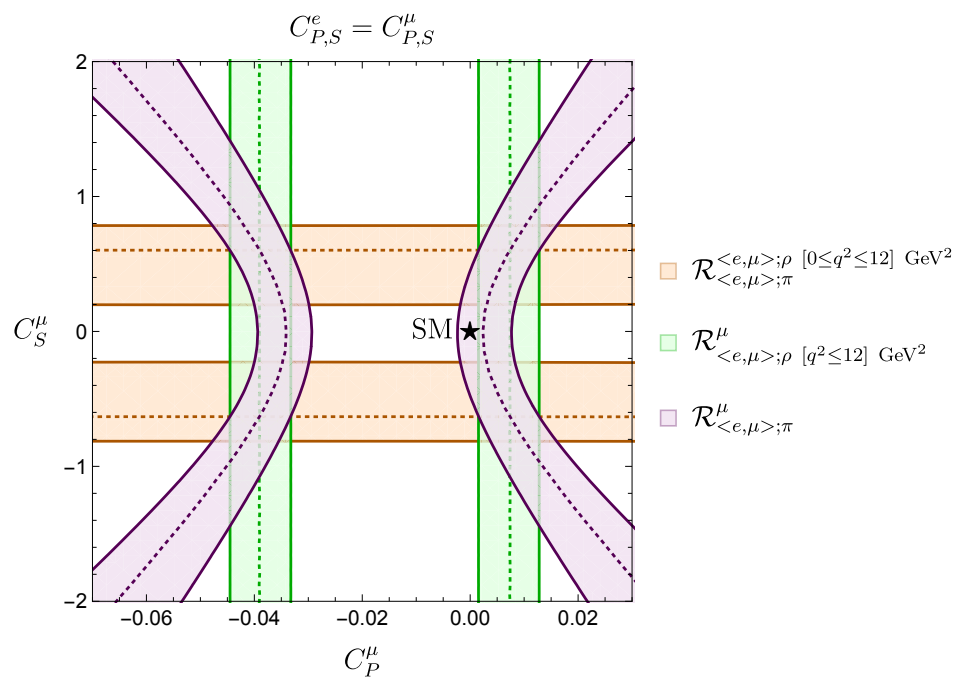

Figure 3: The allowed regions in the $C_{P}^{\mu}-C_{S}^{\mu}$ plane following from the observables $\mathcal{R}_{\langle e, \mu\rangle ; \pi}^{\langle e, \mu\rangle ; \rho\left[0 \leq q^{2} \leq 12\right] \mathrm{GeV}^{2}}, \mathcal{R}_{\langle e, \mu\rangle ; \rho\left[q^{2} \leq 12\right] \mathrm{GeV}^{2}}^{\mu}$ and $\mathcal{R}_{\langle e, \mu\rangle ; \pi}^{\mu}$.

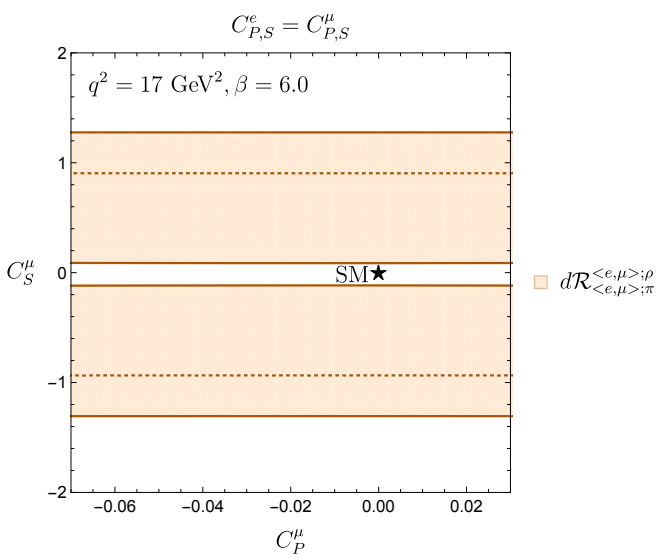

Figure 4: Regions in the $C_{P}^{\mu}-C_{S}^{\mu}$ plane following from the observable $d \mathcal{R}_{\langle e, \mu\rangle ; \pi}^{\langle e, \mu\rangle ; \rho}$ in the large $q^{2}$ regime for $q^{2}=17 \mathrm{GeV}^{2}$.

\section{Determination of $\left|V_{u b}\right|$ and predictions of the branching fractions} for $B^{-} \rightarrow e^{-} \bar{v}_{e}$ and $\bar{B} \rightarrow \rho \tau^{-} \bar{v}_{\tau}$

Having the regions for $C_{P}^{\ell}$ and $C_{S}^{\ell}$ available, we can proceed with the extraction of $\left|V_{u b}\right|$. The strategy introduced at the end of Sec. 1 describes the basic ingredients required for the determination of this CKM matrix element. A more refined procedure, which accounts for possible correlations between our observables, is discussed in [5]. By applying this method, we obtain

$$
\left|V_{u b}\right|=(3.31 \pm 0.32) \times 10^{-3} .
$$

Although in agreement with the value reported by the CKMFitter collaboration [9], presented in Eq. (6), the uncertainty in Eq. (15) is three times bigger. However, our target here is only to illustrate the application of our method which does not assume the SM during the extraction of $\left|V_{u b}\right|$. Future improvements in the precision of our observables will allow us to reduce the 


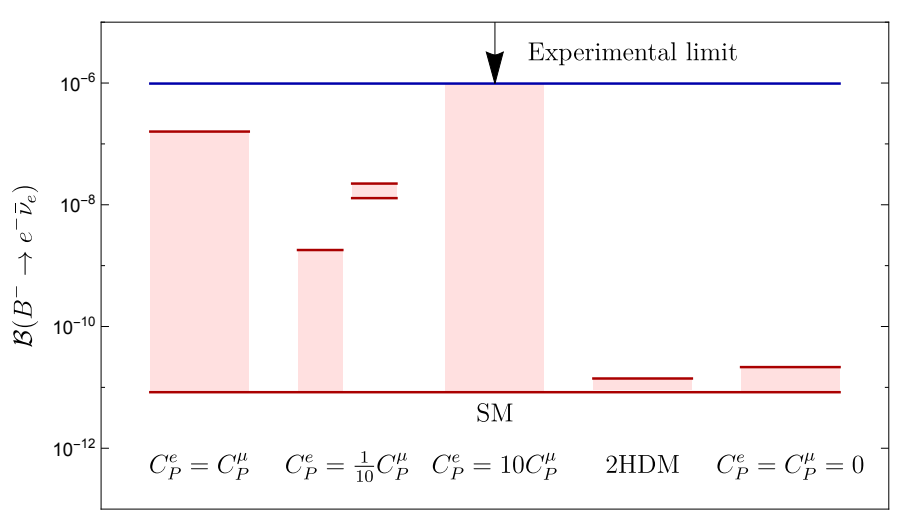

Figure 5: Illustration of the possible enhancement of $\mathcal{B}\left(B^{-} \rightarrow e^{-} \bar{v}_{e}\right)$ for the scenarios discussed in the text. The blue line gives the current experimental upper bound on $\mathcal{B}\left(B^{-} \rightarrow e^{-} \bar{v}_{e}\right)$, whereas the red horizontal line on the bottom represents the $\mathrm{SM}$ value. The red regions indicate the values of the branching ratio that may be obtained.

uncertainty in the CKM matrix element $\left|V_{u b}\right|$.

Besides universal pseudoscalar NP interactions between electrons and muons, other possible scenarios can be considered. We have also explored the cases: $C_{P}^{e}=(1 / 10) C_{P}^{\mu}$ and $C_{P}^{e}=10 C_{P}^{\mu}$. In addition, we included the $2 \mathrm{HDM}$, where the pseudocalar Wilson coefficients for electrons and tau leptons are correlated with $C_{P}^{\mu}$ as

$$
C_{P}^{e}=\frac{m_{e}}{m_{\mu}} \times C_{P}^{\mu}, \quad C_{P}^{\tau}=\frac{m_{\tau}}{m_{\mu}} \times C_{P}^{\mu} .
$$

In these scenarios, our method leads to the value for $\left|V_{u b}\right|$ in Eq. (15). Finally, we have considered the situation where NP affects only the 3rd generation of leptons, i.e. $C_{P}^{\tau} \neq 0$, while $C_{P}^{e}=C_{P}^{\mu}=0$. Here we obtain $\left|V_{u b}\right|=(4.85 \pm 1.03) \times 10^{-3}$, which is closer to the value of $\left|V_{u b}\right|$ following from inclusive determinations $[9,17-24]$.

Finally, we use the ranges for the different Wilson coefficients to make predictions for the yet unmeasured branching ratios $\mathcal{B}\left(B^{-} \rightarrow e^{-} \bar{v}_{e}\right)$ and $\mathcal{B}\left(\bar{B} \rightarrow \rho \tau \bar{v}_{\tau}\right)$. In Fig. 5, the predictions for $\mathcal{B}\left(B^{-} \rightarrow e^{-} \bar{v}_{e}\right)$ are illustrated. We would like to highlight that, for $C_{P}^{e}=10 C_{P}^{\mu}$, our analysis leads to a potential enhancement of $\mathcal{B}\left(B^{-} \rightarrow e^{-} \bar{v}_{e}\right)$ that may even saturate the current experimental bound. An interesting phenomenological prediction in the case $C_{P}^{e}=C_{P}^{\mu}$ is an enhancement by up to four orders of magnitude with respect to the SM value, thereby lying just a factor of 10 below the current experimental upper bound. An analogous effect in the case of $B_{s} \rightarrow e^{+} e^{-}$has been discussed in Ref. [6].

The values for $\mathcal{B}\left(\bar{B} \rightarrow \rho \tau^{-} \bar{v}_{\tau}\right)$ following from our analysis are consistent with the SM picture at the $1 \sigma$ level. As discussed in [5], future measurements of this observable are a powerful tool to distinguish between solutions "1" and "2" in Fig. 2. Our full strategy is summarized in the flow chart in Fig. 6. 


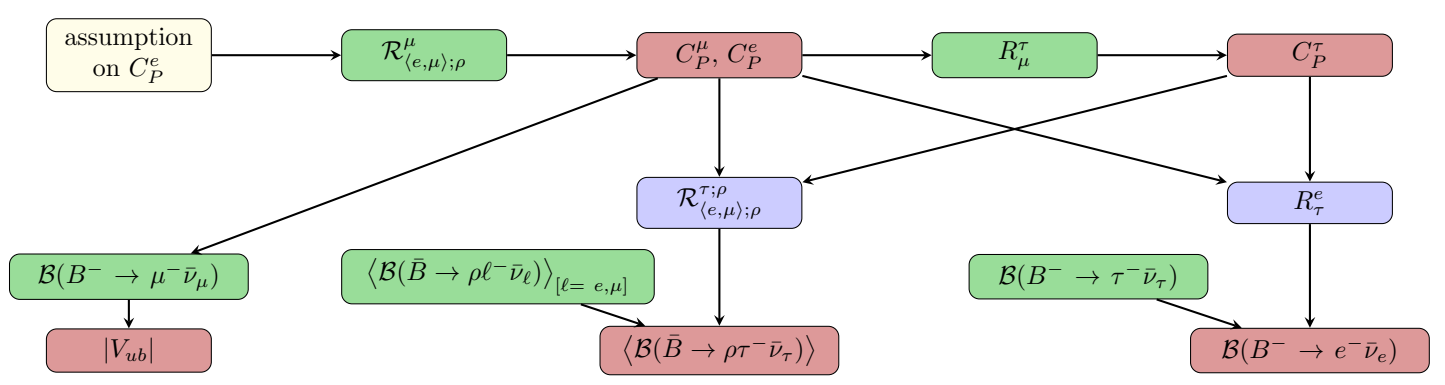

Figure 6: Flowchart illustrating our strategy.

\section{Outlook}

Leptonic and semileptonic decays originating from $b \rightarrow u \ell \bar{v}_{\ell}$ transitions are very interesting and powerful channels to unveil potential NP contributions. They are the counterparts of the $b \rightarrow c \ell \bar{v}_{\ell}$ processes, where recent experimental results for the associated observables $R_{D^{(*)}}$ show effects which may originate from physics beyond the SM.

We have presented a study which analyses the effects of scalar and pseudoscalar particles in leptonic and semileptonic decays arising from the transition $b \rightarrow u \ell \bar{v}_{\ell}$. Central to our analysis is the high sensitivity of the leptonic $B$ decays to the presence of NP pseudoscalar contributions. To take advantage of this feature, we have used the most recent Belle measurement of the branching fraction of the channel $B^{-} \rightarrow \mu^{-} \bar{v}_{\mu}$.

We have developed a strategy with three main goals. Firstly, we have obtained the corresponding short-distance NP contributions, utilizing ratios of leptonic and semileptonic processes where the CKM element $\left|V_{u b}\right|$ cancels. Secondly, we have determined the value of $\left|V_{u b}\right|$, considering simultaneously the presence of NP effects. Finally, we have made predictions for branching fractions of non-yet measured $B$ decays. In particular, we have addressed the decay channels $B^{-} \rightarrow e^{-} \bar{v}_{e}$ and $\bar{B} \rightarrow \rho \tau \bar{v}_{\tau}$, and found NP effects that may be within the reach of the Belle II collaboration and future updates of the LHCb experiment.

Our analysis includes the semileptonic processes $\bar{B} \rightarrow \pi \ell \bar{v}_{\ell}$ and $\bar{B} \rightarrow \rho \ell \bar{v}_{\ell}$ with $\ell=e, \mu, \tau$, where the current experimental data does not provide information on electrons and muons separately. In order to test universality in light leptons of different flavours, it would be desirable that experimental collaborations provide independent measurements for $\ell=e$ and $\ell=\mu$. In addition, a better understanding of the behaviour of the branching fraction of the process $\bar{B} \rightarrow \rho \ell \bar{v}_{\ell}$ requires an update of the corresponding non-perturbative contributions in the high $q^{2}$ regime. Following these lines we will be able to take further advantage of these semileptonic $B$ decays to search for NP. This will complement the ongoing searches in their counterparts $\bar{B} \rightarrow D^{(*)} \ell \bar{v}_{\ell}$, which enter the observables $R_{D^{(*)}}$.

Funding information This research project was supported by the Netherlands Foundation for Fundamental Research of Matter (FOM) programme 156, "Higgs as Probe and Portal", and by the National Organisation for Scientific Research (NWO). 


\section{References}

[1] Y. Amhis et al., Averages of b-hadron, c-hadron, and $\tau$-lepton properties as of summer 2016, Eur. Phys. J. C 77, 895 (2017), doi:10.1140/epjc/s10052-017-5058-4.

[2] F. Svjetlana, Theory status, in The 15th International Workshop on Tau Lepton Physics, Vondelkerk, Amsterdam (2018).

[3] W.-S. Hou, Enhanced charged Higgs boson effects in $B^{-} \rightarrow \tau \bar{\nu}, \mu \bar{v}$ and $b \rightarrow \tau \bar{v}+X$, Phys. Rev. D 48, 2342 (1993), doi:10.1103/PhysRevD.48.2342.

[4] Y. Sakaki, R. Watanabe, M. Tanaka and A. Tayduganov, Testing leptoquark models in $\bar{B} \rightarrow D^{(*)} \tau \bar{v}$, Phys. Rev. D 88, 094012 (2013), doi:10.1103/PhysRevD.88.094012.

[5] G. Banelli, R. Fleischer, R. Jaarsma and G. Tetlalmatzi-Xolocotzi, Decoding (pseudo)scalar operators in leptonic and semileptonic B decays, Eur. Phys. J. C 78, 911 (2018), doi:10.1140/epjc/s10052-018-6393-9.

[6] R. Fleischer, R. Jaarsma and G. Tetlalmatzi-Xolocotzi, In pursuit of new physics with $B_{s, d}^{0} \rightarrow \ell^{+} \ell^{-}$, J. High Energ. Phys. 05, 156 (2017), doi:10.1007/JHEP05(2017)156.

[7] S. Aoki et al., Review of lattice results concerning low-energy particle physics, Eur. Phys. J. C 77, 112 (2017), doi:10.1140/epjc/s10052-016-4509-7.

[8] R. J. Dowdall, C. T. H. Davies, R. R. Horgan, C. J. Monahan and J. Shigemitsu, B-meson decay constants from improved lattice nonrelativistic $Q C D$ with physical $u, d, s$, and c quarks, Phys. Rev. Lett. 110, 222003 (2013), doi:10.1103/PhysRevLett.110.222003.

[9] J. Charles, A. Höcker, H. Lacker, S. Laplace, F. R. Diberder, J. Malclés, J. Ocariz, M. Pivk and L. Roos, $C P$ violation and the CKM matrix: assessing the impact of the asymmetric $B$ factories, Eur. Phys. J. C 41, 1 (2005), doi:10.1140/epjc/s2005-02169-1.

[10] M. Tanabashi et al., Review of particle physics, Phys. Rev. D 98, 030001 (2018), doi:10.1103/PhysRevD.98.030001.

[11] A. Sibidanov et al., Search for $B^{-} \rightarrow \mu^{-} \bar{v}_{\mu}$ decays at the Belle experiment, Phys. Rev. Lett. 121, 031801 (2018), doi:10.1103/PhysRevLett.121.031801.

[12] N. Satoyama et al., A Search for the rare leptonic decays $B^{+} \rightarrow \mu^{+} v_{\mu}$ and $B^{+} \rightarrow e^{+} v_{e}$, Phys. Lett. B 647, 67 (2007), doi:10.1016/j.physletb.2007.01.068.

[13] A. Sibidanov et al., Study of exclusive $B \rightarrow X_{u} \ell v$ decays and extraction of $\left|V_{u b}\right|$ using full reconstruction tagging at the Belle experiment, Phys. Rev. D 88, 032005 (2013), doi:10.1103/PhysRevD.88.032005.

[14] J. A. Bailey et al., $\left|V_{u b}\right|$ from $B \rightarrow \pi \ell v$ decays and (2+1)-flavor lattice $Q C D$, Phys. Rev. D 92, 014024 (2015), doi:10.1103/PhysRevD.92.014024.

[15] K. C. Bowler, J. F. Gill, C. M. Maynard and J. M. Flynn, $B \rightarrow \rho \ell v$ form factors in lattice QCD, J. High Energ. Phys. 05, 035 (2004), doi:10.1088/1126-6708/2004/05/035.

[16] A. Bharucha, D. M. Straub and R. Zwicky, $B \rightarrow V \ell^{+} \ell^{-}$in the Standard Model from lightcone sum rules, J. High Energ. Phys. 08, 098 (2016), doi:10.1007/JHEP08(2016)098.

[17] U. Aglietti and G. Ricciardi, Model for next-to-leading order threshold resummed form factors, Phys. Rev. D 70, 114008 (2004), doi:10.1103/PhysRevD.70.114008. 
[18] U. Aglietti, G. Ferrera and G. Ricciardi, Semi-inclusive B decays and a model for soft-gluon effects, Nucl. Phys. B 768, 85 (2007), doi:10.1016/j.nuclphysb.2007.01.014.

[19] U. Aglietti, F. Di Lodovico, G. Ferrera and G. Ricciardi, Inclusive measure of $\left|V_{u b}\right|$ with the analytic coupling model, Eur. Phys. J. C 59, 831 (2008), doi:10.1140/epjc/s10052-0080817-x.

[20] B. O. Lange, M. Neubert and G. Paz, Theory of charmless inclusive B decays and the extraction of $V_{u b}$, Phys. Rev. D 72, 073006 (2005), doi:10.1103/PhysRevD.72.073006.

[21] S. W. Bosch, B. O. Lange, M. Neubert and G. Paz, Factorization and shapefunction effects in inclusive B-meson decays, Nucl. Phys. B 699, 335 (2004), doi:10.1016/j.nuclphysb.2004.07.041.

[22] S. W. Bosch, M. Neubert and G. Paz, Subleading shape functions in inclusive B decays, J. High Energ. Phys. 11, 073 (2004), doi:10.1088/1126-6708/2004/11/073.

[23] J. R. Andersen and E. Gardi, Inclusive spectra in charmless semileptonic $B$ decays by dressed gluon exponentiation, J. High Energ. Phys. 01, 097 (2006), doi:10.1088/11266708/2006/01/097.

[24] P. Gambino, P. Giordano, G. Ossola and N. Uraltsev, Inclusive semileptonic B decays and the determination of $\left|V_{u b}\right|$, J. High Energ. Phys. 10, 058 (2007), doi:10.1088/11266708/2007/10/058. 